Melinda A. Roberts ${ }^{1}$

\title{
Does the Additional Worth-Having Existence Make Things Better?
}

Let's call the principle that says that the mere addition of the worth-having existence (other things equal) makes things morally better Pareto plus. If we accept Pareto plus, then it seems we should also say that some additions that make at least some person at least a little worse off also may depending on the numbers - make things morally better. I find that latter claim potentially dangerous. As a main focus of an argument why we ought to do something about climate change, I think it moves people not to do anything much about climate change. People won't accept that they or their progeny - or the many, many future people beyond their own progeny should bear a cost, perhaps a significant cost, just to bring ever more future people into existence. But we can't just reject Pareto plus out of hand. For a number of arguments seem to compel us to accept Pareto plus. My goal in this paper is to identify some of the most interesting of those arguments and to show how, in each case, the argument on closer analysis fails. 
Let's call the principle that says that the mere addition of the worth-having existence (other things equal) makes things better Pareto plus. Why is it important to test - to retest - Pareto plus? Why is Pareto plus so dangerous?

If we accept Pareto plus, then it seems we should also accept the idea that there is at least some addition that isn't mere addition - some addition that makes at least some person worse off; perhaps just a little worse off - that also makes things better.

Graph 1: Two Cases: Mere Addition and Not Mere Addition

\begin{tabular}{|c|c|c|c|c|}
\hline & c1 & c2 & c3 & c4 \\
\hline probability & 1 & 1 & 1 & 1 \\
\hline wellbeing & f1 & $\mathrm{f} 2$ & f3 & $\mathrm{f} 4$ \\
\hline+10 & p1, p2...pn & p1, p2...pn, q & p1, p2...pn & p2...pn, q \\
\hline+9 & & & & p1 \\
\hline+0 & $q^{*}$ & & $q^{*}$ & \\
\hline
\end{tabular}

c1 at f1 and c2 at $f 2$ exhaust alternative choices and alternative accessible futures in one case, $c 3$ at f3 and $c 4$ at f4 in the other; boldface indicates people who do or will exist; italics with the asterisk people who will never exist. Wellbeing is raw, unadjusted value, which value in turn determines (I will say) when a future $x$ is better for a person $p$ than a future $y$ is for a person $q^{2}$ The term person includes not just many human beings but many non-human animals as well.

Consider Graph 1 . If $\mathrm{f} 2$ is better than $\mathrm{fl}$, then so is $\mathrm{f} 4$ better than $\mathrm{f} 3$. If adding $\mathrm{q}$ to $\mathrm{f} 2$ adds value to $\mathrm{f} 2$ and makes $\mathrm{f} 2$ better than $\mathrm{fl}$, then it's hard to deny that adding $\mathrm{q}$ to $\mathrm{f} 4$ adds value to 44 and that, the cost to $\mathrm{pl}$ being very small, it adds enough value to make f4 better than f3.

If both Pareto plus and this little extension of Pareto plus are true, then ... I have nothing more to say. But suppose they aren't and we fail to recognize that fact. And suppose we apply the theory we construct on the basis of that mistake to practical issues, including climate change. Then I am concerned that we will leave our audience unmoved to do anything about climate change, indeed moved to do nothing about climate change, and all on the basis of a mistake. For our audience just isn't going to accept that they or, more importantly, their progeny, or, more importantly still, the many, many future people beyond their own progeny, should incur a cost, perhaps a significant cost, just to bring ever more future people into existence.

2 I would thus reject the idea that the "person affecting restriction" means that "trade-offs across people" are "ruled out." Bader (unpublished ms.). 
Thus the hope that it's a person-based theory that will turn out to be true, a theory that will let us say in the first case that $\mathrm{fl}$ is exactly as good as $\mathrm{f} 2$ and in the second case that $\mathrm{f} 3$ is better than $\mathrm{f} 4 .^{3}$ Those implications are I think implications our audience - that is, people asked to sacrifice so that something can be done about climate change - can accept.

At the same time, I recognize that we can't just reject Pareto plus out of hand. For the fact is that a number of arguments seem to compel us to accept Pareto plus. My goal is to identify the most interesting of those arguments and determine for each whether it's as compelling as it seems or has been claimed to be.

I will sketch just a few of those argument and offer replies to each in this paper.

\section{Argument from symmetry}

Consider Graph 2 . The procreative asymmetry states that $\mathrm{fl}$ is worse than $\mathrm{f} 2$ and $\mathrm{c} 1$ is wrong but that $\mathrm{f} 3$ isn't worse than $\mathrm{f} 4$ and $\mathrm{c} 3$ is permissible. More intuitively: it makes things worse to bring the miserable child Meg into existence but doesn't make things better to bring the happy child Holly into existence.

Graph 2: Two Cases: Miserable Child and Happy Child

\begin{tabular}{|c|c|c|c|c|}
\hline & c1 & c2 & c3 & c4 \\
\hline probability & 1 & 1 & 1 & 1 \\
\hline wellbeing & $\mathrm{f1}$ & f2 & f3 & $\mathrm{f} 4$ \\
\hline+10 & & & & Holly \\
\hline+0 & & Meg* & Holly* & \\
\hline-10 & Meg & & & \\
\hline
\end{tabular}

The argument from symmetry challenges that intuition. From Peter Singer: "[W]e do seem to do something bad [and make things worse] if we knowingly bring a miserable being into existence, and if this is so, it is difficult to explain why we do not do something good [why we do not make things better] when we knowingly bring a happy being into existence." But that last point is just Pareto plus. ${ }^{4}$

3 Consider a revised case in which the number of well off people is very great and the cost to the one person is still very small. Depending on the details of the case, the result that the addition of those people makes the future worse will violate certain conditions many find plausible (e.g. Arrhenius 2011). EVNPBI doesn't imply that $\mathrm{f} 3$ is worse than $\mathrm{f} 4$ in the original case and wouldn't generate the parallel result in the revised case. But in virtue of the fact that its necessary condition would be satisfied in all such cases, it does leave the door open for those results (which I don't find implausible) - that is, for other person-based principles to generate the result that the one future is worse than the other.

4 Singer (2011), p. 108. 
Reply: The loss distinction thesis. First, a definition:

A person p sustains a loss in a given future if and only if there is an accessible alternate future that is better for $\mathrm{p}$ (an accessible alternate future in which $\mathrm{p}$ has more wellbeing).

And a concession:

Nonexistence comparability. A future in which a person never exists can be better (or worse) for that person than an alternate future in which a person exists.

Applied here, the view is that Meg sustains a loss at $\mathrm{fl}$ since $\mathrm{f} 2$, where she never exists, is better for her than fl, and Holly sustains a loss at f3, even though she doesn't exist there, since $\mathrm{f} 4$ is better for her than $\mathrm{f} 3$.

Then, the thesis itself:

Loss distinction thesis. For each person p (miserable or happy; existing, future or never existing at all), a loss $\mathrm{p}$ sustains at a future $\mathrm{x}$ has (far-reaching, cross-world) moral significance if and only if $\mathrm{p}$ does or will exist at $\mathrm{x}$.

(Note: If a loss is morally significant, its moral significance isn't arbitrarily contained to any one future or choice; morally significant losses have far-reaching cross-world moral significance. Thus a morally significant loss bears not just on our evaluation of the future in which it is sustained and the choices at that future that give rise to that loss but also on the evaluation of farther flung futures and choices. See Part II below.)

According to the loss distinction thesis, Meg's loss at fl has full, cross-world moral significance and thus counts against $f l$ as compared against $f 2$ and against cl. Holly's loss at f3 has no moral significance and doesn't count against f3 as compared against f4 or against $\mathrm{c} 3$.

Thus a distinction between the two cases, and an account of why it is that Meg's existence makes things worse but Holly's existence doesn't make things better.

The loss distinction thesis may sound a little deontic - or, heaven forbid, commonsensical. But it overlaps and is consistent with a particular way of formulating the person based intuition. 
Expansive very narrow person based intuition (EVNPBI): $\mathrm{c}$ at $\mathrm{x}$ is wrong and a future $\mathrm{x}$ is worse than a future $\mathrm{y}$ only if there is a $\mathrm{p}$ and an alternate accessible future $\mathrm{z}$ such that:

$\mathrm{p}$ does or will exist in $\mathrm{x}$ and $\mathrm{x}$ is worse for $\mathrm{p}$ than $\mathrm{z}$.

This principle is very narrow since, where $\mathrm{p}$ does or will exist in $\mathrm{x}$, it provides only a necessary, not a sufficient, condition on when $\mathrm{x}$ is worse than $\mathrm{y}$ and $\mathrm{c}$ is wrong, and a condition only on x's being worse, not better, than y and on c's being wrong, not c's being permissible.

In another respect, however, this principle is expansive. To determine whether the necessary condition on $\mathrm{x}$ being worse than $\mathrm{y}$ is satisfied, we can't consider only how $\mathrm{p}$ fares in $\mathrm{x}$ as compared against $y$ but must expand the sweep of the analysis to consider as well how $\mathrm{p}$ fares in $\mathrm{x}$ as compared against $z$.

Applied to the asymmetry, EVNPBI implies that f3 isn't worse than f4 and that c3 isn't wrong. And it's consistent with the claim that $\mathrm{fl}$ is worse than $\mathrm{f} 2$ and that $\mathrm{cl}$ is wrong. Thus it leaves room for other person based principles to step in and say that $\mathrm{fl}$ is worse than $\mathrm{f} 2$ and that $\mathrm{cl}$ is wrong.

\section{Argument from moral status of merely possible people}

Many cases - including Addition Plus (Graph 3) - show that the claim that the merely possible, relative to a given future, e.g., the actual future, have moral status. If that's so, and if we accept nonexistence comparability, then it might seem we are compelled to accept Pareto plus.

\section{Graph 3: Addition Plus}

\begin{tabular}{|c|c|c|c|}
\hline & c1 & c2 & c3 \\
\hline probability & 1 & 1 & 1 \\
\hline wellbeing & $\mathrm{f} 1$ & $\mathrm{f2}$ & $\mathrm{f3}$ \\
\hline+11 & & Ann & \\
\hline+10 & Ann & & \\
\hline+5 & & & Ann, Ben \\
\hline+1 & & Ben & \\
\hline+0 & Ben* & & \\
\hline
\end{tabular}


Reply: The loss distinction thesis helps here as well. It recognizes that merely possible people matter morally just as much as you and I do - that we all have the same moral status. But it also says that having moral status just means that, for each of us, some of our losses have moral significance and others don't.

EVNPBI, in contrast to two variations on moral actualism, is again consistent and overlapping with the account of Addition Plus that the Loss Distinction Thesis provides.

\section{Argument from the Mere Addition Principle (MAP)}

Let's call the principle that says that the addition of the worth having existence doesn't (other things equal) make things worse the Mere Addition Principle, or MAP. It might seem that we could accept the seemingly innocuous MAP but reject Pareto plus. In fact, however, we can rework a line of reasoning from John Broome (specifically, his argument against the neutrality intuition) to show that that's not the case.

We start with Broome's own example:

Graph 4: Three Outcome Case (Broome's case)

\begin{tabular}{|c|c|c|c|}
\hline & $\mathrm{c} 1$ & $\mathrm{c} 2$ & $\mathrm{c3}$ \\
\hline probability & 1 & 1 & 1 \\
\hline wellbeing & $\mathrm{f} 1$ & $\mathrm{f} 2$ & $\mathrm{f3}$ \\
\hline+10 & & & Jamie \\
\hline+5 & p1 .. pn & $\mathbf{p 1} \ldots \mathbf{p n}$, Jamie & p1 ... pn \\
\hline+0 & Jamie & & \\
\hline
\end{tabular}

Then, the argument:

1. MAP (assumption)

2. EVNPBI (assumption)

3. $\mathrm{f} 2$ is at least as good as fl (from (1))

4. $\mathrm{f} 3$ is at least as good as fl (from (1))

5. $\mathrm{fl}$ is at least as good as $\mathrm{f} 2$ (from (2))

6. $\mathrm{fl}$ is at least as good as $\mathrm{f} 3$ (from (2))

7. fl is exactly as good as f2 (conceptual truth, (3), (5)) 
8. fl is exactly as good as f3 (conceptual truth, (4), (6))

9. f2 is exactly as good as f3 (conceptual truth, (7), (8))

10. f3 is better than $\mathrm{f} 2$ (same-people Pareto ${ }^{5}$ )

11. Inconsistency (conceptual truths, (9) and (10))

12. If MAP, then EVNPBI is false. (conditional proof, reductio)

13. If EVNPBI is false, then Pareto plus.

14. If MAP, then Pareto plus.

Line (13) isn't obvious. But we can argue for it as follows: If it's not the case that it doesn't make things worse (other things equal) to leave the happy person out of existence, then it makes things worse (other things equal) to leave the happy person out of existence; i.e. it makes things better (other things equal) to bring that person into existence; i.e. Pareto plus.

Reply: We should accept the argument - and the conclusion (14) - but reject MAP. We should, that is, take the position that $\mathrm{f} 2$ is actually worse than $\mathrm{fl}$ (as well as f3). EVNPBI itself implies that $\mathrm{fl}$ is exactly as good as f3; and it leaves the door open for same-people Pareto to say that $\mathrm{f} 2$ is worse than $\mathrm{f} 3$ and thus worse than $\mathrm{fl}$.

Unlike formulations of the person based intuition that don't require the sweeping inquiry, EVNPBI is consistent with the result that $\mathrm{f} 2$ is worse than fl. For its necessary condition for $\mathrm{f} 2$ being worse than $\mathrm{fl}$ is satisfied in this case: since $\mathrm{f} 2$ is worse for $\mathrm{p}$ than $f 3$ is, we are free to take the position that $\mathrm{f} 2$ is after all worse than f1.

\section{Argument from consistency (Independence)}

Compare the Three Outcome Case against the Two Outcome Case.

Applied to this case, EVNPBI implies that $\mathrm{fl}$ is exactly as good as $\mathrm{f} 2$ - which seems right, in this case. But that is inconsistent with what we said before about the Three Outcome Case - it's inconsistent with the position that in that case f2 is worse than fl. To avoid the inconsistency, we should then abandon that position, that is, resurrect MAP and, with MAP, Pareto plus.

5 According to same-people Pareto, when futures fl and $\mathrm{f} 2$ contain exactly the same existing and future people, and $\mathrm{f} 2$ is better for at least some of those people and worse for none of those people, f2 is better than fl. 
Graph 5: Two Outcome Case

\begin{tabular}{|c|c|c|}
\hline & $\mathrm{c1}$ & $\mathrm{c} 2$ \\
\hline probability & 1 & 1 \\
\hline wellbeing & $\mathrm{f} 1$ & $\mathrm{f} 2$ \\
\hline+10 & & \\
\hline+5 & $\mathbf{p 1 ~ \ldots . ~ p n ~}$ & $\mathbf{p 1} \ldots$ pn, Jamie \\
\hline+0 & Jamie* & \\
\hline
\end{tabular}

Reply: More precise nomenclature will clarify that $\mathrm{fl}$ and the $\mathrm{f} 2$ in the Two Outcome Case are distinct from $\mathrm{fl}$ and the $\mathrm{f} 2$ in the Three Outcome Case. Once that is done, the inference to $\mathrm{f} 3$ is better than $\mathrm{fl}$ in the Three Outcome Case fails (and we avoid inconsistency or any violation of independence).

The distinction in futures from the one case to the other isn't just coincidental; we can't resurrect the original problem by leaving everything else about the two cases alone and then stipulating that the fl (f2) in the one case is identical to fl (f2) in the other case. Why not?

Accessibility axiom: If $\mathrm{y}$ is an accessible alternative future relative to $\mathrm{x}$, then necessarily $\mathrm{y}$ is an accessible alternative future relative to $\mathrm{x}$.

In other words, if $y$ is accessible to $x$ in any case, then y's existence as such a future is built into the very identity of $x$, possible futures, or worlds, having all their features necessarily.

\section{Argument from simple nonidentity}

In this case, $\mathrm{cl}$ and $\mathrm{c} 2$ exhaust the agents' choices and $\mathrm{fl}$ and f2 the alternative accessible futures; $p$ and $q$ are (of course) distinct.

Graph 6: Simple Nonidentity

\begin{tabular}{|c|c|c|}
\hline & $\mathrm{c} 1$ & $\mathrm{c} 2$ \\
\hline probability & 1 & 1 \\
\hline wellbeing & $\mathrm{f} 1$ & $\mathrm{f} 2$ \\
\hline+10 & & $\mathbf{q}$ \\
\hline+8 & $\mathbf{p}$ & \\
\hline+0 & $q^{*}$ & $p^{*}$ \\
\hline
\end{tabular}


It's standardly said about this case (by Parfit and many others, complying with a dominance principle ${ }^{\sigma}$ ) that $\mathrm{fl}$ is worse than $\mathrm{f} 2$ and that $\mathrm{cl}$ is wrong. Let's call those claims together the standard view. If the standard view is true, then that would seem to suggest - and I admit that there's a leap in the argument at this point ${ }^{7}$ - that f3 is worse than $\mathrm{f} 4$ in the following case (Simple Addition; Graph 7). Still, that leap of logic seems at least plausible since it seems that any plausible theory that implies that fl is worse than $\mathrm{f} 2$ though worse for no one also will imply that $\mathrm{f} 3$ is worse than $\mathrm{f} 4$ though worse for no one.

Graph 7: Simple Addition

\begin{tabular}{|c|c|c|}
\hline & $\mathrm{c3}$ & $\mathrm{c4}$ \\
\hline probability & 1 & 1 \\
\hline wellbeing & $\mathrm{f3}$ & $\mathrm{f} 4$ \\
\hline+10 & & $\mathbf{p}$ \\
\hline+8 & & \\
\hline+0 & $p^{*}$ & \\
\hline
\end{tabular}

Reply: We should reject the standard view, accept that $\mathrm{fl}$ is exactly as good as $\mathrm{f} 2$ and that $\mathrm{f} 3$ is exactly as good as $\mathrm{f} 4$ - and that $\mathrm{cl}-\mathrm{c} 4$ are all permissible.

Notably, this is not to say that we should also accept that the choice of depletion is permissible or that the future that includes depletion is exactly as good as the future that includes conservation. Ditto the "do nothing" choice in respect of climate change. The simple nonidentity problem is very different from the probabilistic form of the nonidentity problem, which includes depletion, risky policy, climate change, historical injustice, pleasure pill, slave child, etc. (See Part VII below.)

\section{Argument from additivity}

We need additivity to insure correct logical features for the betterness relation (e.g., transitivity; no cycling) and also to make the evaluation process efficient (to insure the consistency of sequential pairwise comparisons). Additivity implies Pareto plus.

Reply: I suspect we can preserve the correct logical features - including transitivity and symmetry - without additivity. In any case, additivity doesn't imply Pareto plus. What we add up, under additivity, needn't be raw, unadjusted wellbeing levels. We can instead add up wellbeing adjusted to reflect our existential values.

6 Arrhenius (2000).

7 Person-affecting principles discussed by Bader and Holtug avoid this leap of logic. See Bader (unpublished ms.) and Holtug (2010). 
Thus Broome argues that the personal good - what is added up to determine the overall (general) good of a world - can be understood to reflect the values of equality and fairness. Setting those values aside, I propose that personal good can be understood to reflect - that wellbeing can be adjusted to reflect - our existential values.

To make that proposal work in the Three Outcome Case (just to take one example), we would understand the personal good level for $\mathrm{p}$ in f3 where p's wellbeing is maximized to be zero and the personal good level for $\mathrm{p}$ in $\mathrm{f} 2 \mathrm{p}$ 's wellbeing is avoidably lower to fall in the negative range (even though wellbeing itself is positive). In contrast, in the Two Outcome Case, the personal good level for $\mathrm{p}$ in $\mathrm{f} 2$ would just be zero.

\section{Argument from better (greater) chance}

A better, that is, greater, chance of existence makes things morally better. At least: it's incontrovertible that Harry's better chance of existence under cl makes cl permissible. Therefore so must the fact of existence make things morally better.

Graph 8: Better Chance Case

\begin{tabular}{|c|c|c|c|c|}
\hline & \multicolumn{2}{|c|}{ c1: take fertility pill } & \multicolumn{2}{c|}{ c2: take aspirin } \\
\hline probability & 0.1 & 0.9 & 0.0001 & 0.9999 \\
\hline wellbeing & $\mathrm{f1}$ & $\mathrm{f2}$ & $\mathrm{f3}$ & $\mathrm{f4}$ \\
\hline+10 & & & Harry & \\
\hline+8 & Harry & & & \\
\hline+0 & & Harry* & & Harry $^{*}$ \\
\hline
\end{tabular}

Reply: We should agree that Harry's better chance of existence under cl makes the otherwise wrong cl permissible.

That seems a core component of the person based intuition. But it's not one that EVNPBI itself expresses. The question is whether we can revise EVNPBI in a way that makes the point that the better chance at existence makes things better - at least in the sense that it makes the otherwise wrong $\mathrm{cl}$ permissible - but avoids the further point, a point entirely at odds with the person based idea, that existence doesn't make things better. If we can't do that, we have an intuition at odds with itself.

Reply: We can do that. First a definition: 
Where a choice $\mathrm{c}$ made at a future $\mathrm{x}$ creates a probability $\mathrm{n}$ that $\mathrm{p}$ will have the wellbeing level $(w b)$ that $\mathrm{x}$ in fact assigns to $\mathrm{p}$, we can say that $x$ 's probable value (PV) for $p$ under $c$ is $\mathrm{n}(\mathrm{wb})$.

(Note: We might naturally want to reference the fact that $\mathrm{cl}$ creates more expected value for Harry than c2 does to explain cl's permissibility. But other cases show that approach fails by virtue of the fact that expected value can be greatly inflated by a low probability of a very wonderful outcome. ${ }^{8}$ )

And then:

Expansive Very Narrow Person Based Intuition + Probable Value $(E V N P B I+P V)$ : A future $\mathrm{x}$ is worse than a future $\mathrm{y}$ only if there is a $\mathrm{p}$ and an alternate accessible future $\mathrm{z}$ such that:

$\mathrm{p}$ does or will exist in $\mathrm{x}$ and $\mathrm{x}$ is worse for $\mathrm{p}$ than $\mathrm{z}$.

$\mathrm{c}$ at $\mathrm{x}$ is wrong, only if there is a person $\mathrm{p}$ and an alternate choice $\mathrm{c}^{\prime}$ at an alternate accessible future $y$ such that

$\mathrm{p}$ does or will exist in $\mathrm{x}$ and $\mathrm{x}$ is worse for $\mathrm{p}$ than $\mathrm{y}$ and $\mathrm{PV}$ of $\mathrm{c}$ at $\mathrm{x}$ for $\mathrm{p}<\mathrm{PV}$ of $\mathrm{c}^{\prime}$ at $\mathrm{y}$ for $\mathrm{p}$.

To sum up: according to EVNPBI+PV, Harry's better chance under $\mathrm{cl}$ at $\mathrm{fl}$ makes $\mathrm{cl}$ permissible (when it would otherwise be wrong). But, also according to EVNPBI+PV, $\mathrm{c} 2$ is permissible. Moreover, nothing in EVNPBI+PV suggests that Harry's better chance of existence embedded in fl under cl makes fl better than $\mathrm{f} 3$ makes, that is, make f3 worse than fl. So we are free to retain same-people Pareto and insist that $\mathrm{fl}$ is worse than $\mathrm{f} 3$.

We are free, that is, to insist, more generally, that, where $\mathrm{x}$ and $\mathrm{y}$ assign to $\mathrm{p}$ the same wellbeing level but chances of $p$ getting that wellbeing level - e.g. chances of $p$ coming into existence at that level - are greater at $\mathrm{x}$ than at $\mathrm{y}, \mathrm{x}$ is exactly as good as $\mathrm{y}$.

Compare cases that ground the probabilistic (non-simple) versions of the nonidentity problem, e.g., depletion, risky policy, "do nothing” choice on climate

8 Roberts (unpublished ms.). I owe this point to Dean Spears. 
change, historical injustice, slave child, pleasure pill etc. In those cases it's clear we want to say the choice is wrong. And in those cases - on closer inspection - we should understand that the apparent victim - that is, the victim - doesn't have a better chance of existence under the suspect choice than he or she does under some (indeed many) relevant alternative choices. ${ }^{9}$ Thus p's chances of existence are no greater under the choice of the pleasure pill than under the choice of the aspirin (the pleasure pill isn't a fertility pill); no greater under the choice of depletion, risky policy, than under the choice of conservation, safe policy. ${ }^{10}$

Compare the simple nonidentity problem (Graph 6 above), e.g., the case involving a genetic disorder that can't accessibly be mitigated or cured, cases in which the apparent victim can't be made better off (the agents lack the ability, the physical laws governing the world lack the flexibility), that is, in which the only alternative accessible future is one in which a nonidentical but better off child exists in place of the one.

\section{Other issues}

(1) Does EVNPBI+PV violate complementarity (Spears, unpublished ms.)? According to complementarity, it's better to assign (a "prospect" is better if it assigns) a higher probability of existence to the better off person than a lower probability of existence to the worse off person. By implication, it's better to assign a higher probability to the one person's existing and being better off than a lower probability to the one person's existing and being worse off. A case that would have those features is the fertility case amended so that the fertility pill is actually safer from the perspective of the future child than the aspirin is (perhaps now the aspirin causes brain bleeds).

Reply: No reason to think EVNPBI+PV violates complementarity. It avoids the implication that $\mathrm{c} 2$ at $\mathrm{f} 3$ is permissible. And we can still say that $\mathrm{fl}$ is exactly as good as $\mathrm{f} 2$ is exactly as good as $\mathrm{f} 4$ and that all are better than $\mathrm{f} 3$.

\footnotetext{
9 That won't necessarily be the choice the agents would have made instead; the choice agents would have made in a given case may well make things worse for all involved. The choice we should compare against is instead one that agents could have made (whether they would have or not). Thus in the pleasure pill case we can add that, had the couple not taken the pleasure pill, they would, in a snit, not have conceived any child at all. That new fact is, however, a red herring - it doesn't change the analysis. The important thing is what the couple could have done. They could have taken the aspirin and conceived a child on just the same schedule on which they could have taken the pleasure pill and conceived a child. Note that in both cases, prior to choice, there exist a vast numbers of ways of implementing the particular choice, whether the choice to take the pleasure pill or the choice to take the aspirin. That's why under the pleasure pill choice as well any particular child's coming into existence is very low.

10 That is, the probable value the choice of the pleasure pill creates for the apparent victim isn't higher, but rather lower, than the choice of the aspirin creates for that same person.
} 
(2) Does EVNPBI+PV violate anonymity (as described by Spears (unpublished ms.), or by Thomas (unpublished ms.), or by Bader (unpublished ms.), in the form of impartiality? Consider the following case:

Graph 9: Identity Matters

\begin{tabular}{|c|c|c|c|}
\hline & c1 & c2 & c3 \\
\hline probability & 1 & 1 & 1 \\
\hline wellbeing & $\mathrm{f} 1$ & $\mathrm{f2}$ & $\mathrm{f3}$ \\
\hline+10 & & & $\mathbf{q}$ \\
\hline+5 & $\mathbf{p}$ & $\mathbf{q}$ & $p^{*}$ \\
\hline+0 & $q^{*}$ & $p^{*}$ & \\
\hline
\end{tabular}

Anonymity implies that $\mathrm{fl}$ is exactly as good as $\mathrm{f} 2$. But EVNPBI+PV implies that $\mathrm{fl}$ is exactly as good as f3 and same-people Pareto that f3 is better than f2. Thus, fl is better than $\mathrm{f} 2$, which violates anonymity.

Reply: We should accept the violation - that is, we should reject anonymity. Doing so preserves the highly intuitive EVNPBI+PV. Moreover, rejecting anonymity enables us to avoid implausible results in many infinite population problems. See e.g. Thomas's discussion of Welfarist Anonymity and Population Separability in the context of the case of "Steve." Thomas (unpublished ms.). The range of infinite population we shall ultimately need to address is considerable - and thus a discussion we shall need to reserve for another paper.

\section{Conclusion}

In this paper, I have addressed a handful of interesting and often compelling arguments in favor of Pareto plus, the idea that other things equal the additional worthhaving existence makes things morally better. I have argued that none of those arguments in fact succeeds in establishing that conclusion. The upshot is that it is beginning to look like we face no compelling reason not to reject Pareto plus if that is what we want to do. That result has practical importance. We retain credibility when we put forward analyses in support of the view that substantial sacrifice is both necessary and desirable for purposes of addressing climate change - that agents today ought to make, and be willing to impose on others, such sacrifice in order to make things go better for future generations. But we may lose credibility when we argue that agents ought not just to take steps to make things better for future generations but also take steps -- make, and impose on others, vast additional sacrifice - so that ever more future people can be brought into existence. If theory and credibility align - one allowing us to reject Pareto plus, the other simply a matter 
of people's attitudes toward the sacrifices they may reasonably be asked to make the specter of our utter failure to address climate change in the very near future may begin to recede.

\section{References}

Arrhenius, Gustaf (2000), “An Impossibility Theorem for Welfarist Axiologies,” Economics and Philosophy 16: 247-266.

Arrhenius, Gustaf (2011), “The Impossibility of a Satisfactory Population Ethics,” World Scientific Review 20(54): 1-26.

Bader, Ralf (forthcoming), "Person-Affecting Utilitarianism," in T. Campbell, K. Bykvist, G. Arrhenius, E. Finneron-Burns (eds.), The Oxford Handbook of Population Ethics. Oxford: Oxford University Press.

Holtug, Nils 2010. Persons, Interests, and Justice, Oxford University Press.

Roberts, Melinda A. (unpublished ms.), The Existence Puzzles.

Singer, Peter (2011), Practical Ethics (3rd. ed.), Cambridge University Press.

Spears, Dean (unpublished ms.), "Probabilistic Future People”.

Thomas, Teruji (forthcoming), “Separability,” in T. Campbell, K. Bykvist, G. Arrhenius, E. Finneron-Burns (eds.), The Oxford Handbook of Population Ethics. Oxford: Oxford University Press. 